\title{
Gravitational anomalies and one-dimensional behavior of black holes
}

\author{
Bibhas Ranjan Majhi ${ }^{\mathrm{a}}$ \\ Department of Physics, Indian Institute of Technology Guwahati, Guwahati 781039, Assam, India
}

Received: 26 October 2015 / Accepted: 25 November 2015 / Published online: 8 December 2015

(c) The Author(s) 2015. This article is published with open access at Springerlink.com

\begin{abstract}
It has been pointed out by Bekenstein and Mayo that the behavior of the black hole's entropy or information flow is similar to information flow through one-dimensional channel. Here I analyze the same issue with the use of gravitational anomalies. The rate of the entropy change $(\dot{S})$ and the power $(P)$ of the Hawking emission are calculated from the relevant components of the anomalous stress tensor under the Unruh vacuum condition. I show that the dependence of $\dot{S}$ on the power is $\dot{S} \propto P^{1 / 2}$, which is identical to that for the information flow in a one-dimensional system. This is established by using the $(1+1)$-dimensional gravitational anomalies first. Then the fact is further bolstered by considering the $(1+3)$-dimensional gravitational anomalies. It is found that, in the former case, the proportionality constant is exactly identical to the one-dimensional situation, known as Pendry's formula, while in the latter situation its value decreases.
\end{abstract}

The fact that the black hole has one-dimensional nature as long as the entropy flow is concerned has already been broadcast by Bekenstein and Mayo [1]. This is a complementary statement to the "holographic principle" [2]. The idea was to calculate the rate of entropy change for the emission spectrum from the horizon. In this approach the entropy current was defined by the entropy density multiplied by the group velocity of the emitted particle. It turns out that the rate of entropy flow is proportional to the square root of the energy current or power of the emitted spectrum. The same nature also occurs for an one-dimensional system, except the proportionality constant is different. In this analysis the spacetime was taken to be the $(1+3)$-dimensional Schwarzschild black hole. Recently, the analysis has been extended to arbitrary $D$-dimensional Schwarzschild spacetime [3] and the conclusion is the same, except that the proportionality constant now depends on the dimension of the spacetime.

\footnotetext{
a e-mail: bibhas.majhi@iitg.ernet.in
}

In this letter, the one-dimensional behavior of the black hole will be explored in the context of gravitational anomalies. The roles of anomalies have already been explored in different contexts of gravitational physics. It has already been observed that the gravitational anomalies can explain the Hawking effect [4-9]. The correct expression for the Hawking flux was derived from the anomalous stress tensor with a suitable choice of the boundary condition (see for details [8]). Recently, the modifications to the constitutive relations for a fluid were found in the presence of anomalies [10-13]. But so far, no one discussed any connection between the gravitational anomalies and the one-dimensional behavior of the black holes. Here I shall precisely address this issue. I shall show that the existence of anomalies at the quantum level can explain this precise property of the emission from the horizon.

The organization of the letter is as follows. We shall begin our discussion with the near horizon effective theory which is $(1+1)$-dimensional and hence the theory is accompanied by the two-dimensional anomalies. Solutions of the anomalous equations will give the various components of stress tensor. Using them in the Gibbs-Duhem relation [14], the rate of the entropy change of the emitted particle will be calculated. We will see that it is, like the one-dimensional radiation, proportional to the square root of the power of the emission spectrum. Finally, this fact will be bolstered by extending the same approach for the $(1+3)$-dimensional theory in the presence of gravitational anomalies.

We start our analysis with the near horizon effective $(1+$ 1)-dimensional theory where the two-dimensional anomalies appear. The whole discussion will be confined within the metrics which are asymptotically flat at infinity. The reason for the restriction will become clear later on. Now the question is why we are interested in the two-dimensional case, although the full spacetime is not $(1+1)$-dimensional. The reason is as follows. One of the interesting facts of black hole spacetimes is that they are effectively $(1+1)$-dimensional near the 
event horizon $[5,6]$. This can be seen in the following way. For simplicity, consider a scalar field action with mass and interaction terms in the case of a full black hole spacetime. The action, when expanded and when the near horizon limit has been taken, reduces to a free action for a collection of scalar field modes with the $(1+1)$-dimensional background of the form

$\mathrm{d} s^{2}=-f(r) \mathrm{d} t^{2}+\frac{\mathrm{d} r^{2}}{f(r)}$,

where the horizon $r=r_{0}$ is determined by the equation $f\left(r_{0}\right)=0$. Therefore, it turns out that the theory, near the horizon, is effectively a two-dimensional conformal theory, because the mass term or any other interaction term vanishes. Moreover, the effective metric in this region is taken to be that given by Eq. (1). Hence, here the theory is dominated by this metric (for more details, see [15]). It appeared that such a fact is very useful to study several features of black holes. For instance, the Hawking effect has been discussed via two interesting approaches: one is by the gravitational anomaly method [5-9] and the other is by the tunneling approach [16-20]. In the first approach, it is argued that since the near horizon theory is two-dimensional, the theory must accompanied by gravitational anomalies (two-dimensional). These are manifested through the non-conservation of the energymomentum tensor and non-vanishing of the trace of it. Then the $(t, r)$ component of the stress tensor, obtained by solving the anomaly equations under the metric (1), with the Unruh boundary condition leads to the correct expression for the emission flux from the horizon. In the other approach, one considers the tunneling of a particle to be happening along a radial path. So no angular part will contribute and the metric can be considered of the form given by (1). Finally the correct Hawking temperature is identified by calculating the tunneling probability [16-19] or the emission spectrum [20]. Also, the entropy has been calculated using the tunneling modes [21]. So from the above evidence it is clear that the effective metric plays a central role in black hole thermodynamics. Furthermore, the Hawking temperature has been obtained by the global embedding approach of the effective metric of the form (1) instead of the full metric [22]. Hence, we feel that it might be a hint in favor of the one-dimensional (which means one space dimension) nature of the black hole emission.

From the above discussion, one might think that the $(1+1)$ dimensional gravitation anomalies can shed some light on the one-dimensional entropy flow nature. I shall show in the following that this is indeed possible. The two-dimensional (which means one time and one space coordinate) gravitational anomalies are manifested by the non-conservation and non-zero value of the trace of the energy-momentum tensor [23-26]: $\nabla_{b} T^{a b}=c_{g} \bar{\epsilon}^{a b} \nabla_{b} R ; \quad T_{a}^{a}=c_{w} R$

where $c_{g}$ and $C_{w}$ are anomaly coefficients and $R$ is the twodimensional Ricci scalar. The expressions for the components of the energy-momentum tensor, under the background (1), turn out to be [12]

$$
\begin{aligned}
& T_{u u}=\frac{2 c_{g}+c_{w}}{4}\left(f f^{\prime \prime}-\frac{f^{\prime 2}}{2}\right)+C_{u u} ; \\
& T_{v v}=-\frac{2 c_{g}-c_{w}}{4}\left(f f^{\prime \prime}-\frac{f^{\prime 2}}{2}\right)+C_{v v} ; \\
& T_{u v}=-\frac{c_{w}}{4} f R=\frac{c_{w}}{4} f f^{\prime \prime} ;
\end{aligned}
$$

where $C_{u u}$ and $C_{v v}$ are integration constants. The above are expressed in null coordinates: $u=t-r_{*}$ and $v=t+r_{*}$ with $\mathrm{d} r_{*}=\mathrm{d} r / f(r)$. Now since we are interested to the emitted particles from the horizon; i.e. the Hawking radiated particle, one must impose the Unruh vacuum [8] to fix the integration constants. This is defined by the conditions $T_{u u}=0$ close to the horizon, $r \rightarrow r_{0}$, while $T_{v v}=0$ at asymptotic infinity, $r \rightarrow \infty$. Imposition of the boundary conditions leads to

$C_{u u}=\left(2 c_{g}+c_{w}\right) 2 \pi^{2} T_{0}^{2} ; \quad C_{v v}=0 ;$

where $T_{0}=f^{\prime}\left(r_{0}\right) / 4 \pi$ is the Hawking temperature. Next we shall define the energy density and the entropy density by the above components.

Now it is well known that the time-time component of the stress tensor, i.e. $T_{t}^{t}$, is related to the energy density $(\epsilon)$, while the radial-radial component; i.e. $T_{r}^{r}$, is related to the pressure $(p)$ of the emitted radiation. So to determine these quantities one has to express (3) in the original $(t, r)$ coordinates. In these coordinates the components of $T_{a b}$ reduce to the following forms:

$$
\begin{aligned}
T_{t}^{t}= & -\frac{1}{f}\left(T_{u u}+T_{v v}+2 T_{u v}\right)=-\frac{c_{w}}{4}\left(4 f^{\prime \prime}-\frac{f^{\prime 2}}{f}\right) \\
& -\frac{C_{u u}+C_{v v}}{f} ; \\
T_{r}^{r}= & \frac{1}{f}\left(T_{u u}+T_{v v}-2 T_{u v}\right)=-\frac{c_{w}}{4} \frac{f^{\prime 2}}{f}+\frac{C_{u u}+C_{v v}}{f} .
\end{aligned}
$$

If the energy density and the entropy density are denoted by $\epsilon$ and $s$, respectively, then the energy current is given by $\epsilon^{a}=\epsilon u^{a}$, while the entropy current turns out to be $s^{a}=$ $s u^{a}$ where $u^{a}$ is the velocity of the emitted radiation. Now if we want to calculate these quantities in the co-moving frame, then the components of the velocity for the metric (1) are $u^{a}=(1 / \sqrt{f}, 0)$. Hence only the time components of both the currents are non-zero. Now the rate of change of energy; i.e. the power $(P)$, near the horizon, as measured from infinity, is 


$$
\begin{aligned}
P & =\left.\epsilon^{t}\right|_{r \rightarrow \infty}=-\left.T_{t}^{t} u^{t}\right|_{r \rightarrow \infty}=C_{u u}+C_{v v} \\
& =\left(2 c_{g}+c_{w}\right) 2 \pi^{2} T_{0}^{2} .
\end{aligned}
$$

To calculate the rate of change of the entropy, we need to find the entropy density first. Using the Gibbs-Duhem relation, $T_{0} s=\epsilon+p$, one finds the entropy density,

$s=\frac{\epsilon+p}{T_{0}}=\frac{-T_{t}^{t}+T_{r}^{r}}{T_{0}}$.

Therefore, similar to the power, the rate of change of the entropy, as measured from infinity, turns out to be

$\dot{S}=\left.s u^{t}\right|_{r \rightarrow \infty}=\frac{2\left(C_{u u}+C_{v v}\right)}{T_{0}}=\left(2 c_{g}+c_{w}\right) 4 \pi^{2} T_{0}$.

Note that to calculate the total rate of change of any quantity we need to integrate the corresponding density by the space volume for the unit time. In the present analysis we first calculate them near the horizon where the space dimension is one and so the rate of change of them turn out to be the density multiplied by the velocity; i.e. the current. Next they have to be measured from infinity where $f^{\prime}$ and $f^{\prime \prime}$ vanish as for an asymptotic flat spacetime the form of the metric coefficient is of the form $f(r)=1+a_{0} / r+\mathcal{O}\left(1 / r^{2}\right)$. This has precisely been done in the above calculations. Next, combining (6) and (8) we find the relation between $\dot{S}$ and $P$ as

$\dot{S}=\frac{2 P}{T_{0}}=\left[8 \pi^{2}\left(2 c_{g}+c_{w}\right) P\right]^{1 / 2}$.

The first equality is exactly the same as obtained earlier for a one-dimensional thermal radiation of photon (see Eq. (4) of [1]). The second equality shows that $\dot{S}$ is proportional to square root of the power. This is again identical in nature to the one-dimensional case. The proportionality constant, of course, depends on the value of the anomaly coefficients. It has been observed earlier that the $T_{t}^{r}$ component leads to the correct value of the Hawking flux for either $c_{w}=$ $1 / 24 \pi$ and $c_{g}=0$ (only a trace anomaly exists) [4] or $c_{w}=$ $1 / 48 \pi$ and $c_{g}=1 / 96 \pi$ (both trace and diffeomorphism anomalies exist) [5-9]. In any of the two cases we have $2 c_{g}+$ $c_{w}=1 / 24 \pi$. Interestingly, then the proportionality constant turns out to be $(\pi / 3)^{1 / 2}$, which is identical to that for the one-dimensional radiation [1] and hence Eq. (9) is exactly identical to Pendry's result [27]. The reason is the following. Remember that the above analysis is valid in the near horizon limit, and in this region the effective potential of the black hole spacetime is very small. Therefore the effect of it can be neglected and hence the radiation is pure thermal. This has exactly been accounted for in the above analysis and one can justify this by finding the $T_{t}^{r}$ component at infinity (which is the Hawking flux) from (3). Since Pendry's work is also based on the thermal radiation, it is not surprising that the present case exactly matches with that of Pendry. Of course, if one considers the gray body factor in the emission spectrum from the horizon, then these two analyses must not give identical results.

So far the one-dimensional nature of black holes has been discussed based on the near horizon effective theory. Now in the followoing, I shall use the $(1+3)$-dimensional gravitational anomaly and show that the radiation from the horizon has a similar nature. In the literature, the expressions for the components of the renormalized stress tensor corresponding to the trace anomaly in the case of a Schwarzschild black hole occur. Without introducing much details, let me just borrow the expressions. The stress tensor in the Unruh vacuum, in the asymptotic infinity limit $r \rightarrow \infty$, is given by [28,29],

$T_{b}^{a}=\frac{L}{4 \pi r^{2}}\left(\begin{array}{cccc}-1 & -1 & 0 & 0 \\ 1 & 1 & 0 & 0 \\ 0 & 0 & 0 & 0 \\ 0 & 0 & 0 & 0\end{array}\right)$,

where $L$ is known as the luminosity and its value is $L=$ $C_{0} / \pi M^{2}, M$ is the mass of the Schwarzschild black hole. The value of the constant $C_{0}$, obtained by two different methods, is $[30,31]$

$C_{0}= \begin{cases}2.197 \times 10^{-4}, & \text { by geometric optics } \\ 2.337 \times 10^{-4}, & \text { by numerical estimation. }\end{cases}$

So in this case, the energy density and pressure are given by $\epsilon=-T_{t}^{t}=L / 4 \pi r^{2}=16 C_{0} T_{0}^{2} / r^{2}$ and $p=T_{r}^{r}=$ $L / 4 \pi r^{2}=16 C_{0} T_{0}^{2} / r^{2}$, respectively, where $T_{0}=1 / 8 \pi M$ is the Hawking temperature. Therefore, the entropy density is $s=(\epsilon+p) / T_{0}=32 C_{0} T_{0} / r^{2}$. As earlier, to find the rate of change of these quantities we need to integrate them by the space volume per unit time. This in a co-moving frame is given by $4 \pi r^{2} u^{a}$. Now since $u^{a}=(1 / \sqrt{f}, 0,0,0)$ the value of the unit time space volume at infinity is $4 \pi r^{2}$. Hence the power and the rate of change of the entropy of the emitted spectrum are

$P=4 \pi r^{2} \epsilon=64 \pi C_{0} T_{0}^{2}$;

$\dot{S}=4 \pi r^{2} s=128 \pi C_{0} T_{0}$.

Combining these two we obtain an identical relation to that of the earlier effective two-dimensional case: $\dot{S}=2 P / T_{0}$ (see the first equality of Eq. (9)). Now using the expression for $T_{0}$ in terms of $P$ from the first relation of (12) we find

$\dot{S}=16 C_{0}^{1 / 2}(\pi P)^{1 / 2}$.

Here also we get a similar dependence of rate of change of entropy flow on the power like the one-dimensional one. The only difference is the value of the proportionality constant. In the one-dimensional case its value is $(\pi / 3)^{1 / 2}$, whereas in $(1+3)$ dimensions the present analysis shows that the value is 
$16 C_{0}^{1 / 2} \pi^{1 / 2}= \begin{cases}0.237 \times \pi^{1 / 2}, & \text { by geometric optics, } \\ 0.245 \times \pi^{1 / 2}, & \text { by numerical estimation. }\end{cases}$

So (13) is 41-42\% of the one-dimensional situation.

Before finishing the discussion, let me point out that Bekenstein and Mayo [1] got a different proportionality constant in the case of the four-dimensional Schwarzschild black hole. Their value is $0.087 \times \pi^{1 / 2}$. So in my analysis the rate of change of the entropy increases by $2.72-2.82$ times compared to that obtained in [1]. This discrepancy is due to the following fact. In $(1+3)$ dimensions, the anomaly equations cannot be solved exactly and hence the value of the constant $C_{0}$ is not exact. However, the value determined by Bekenstein and Mayo is based on the numerical estimations done by Page $[32,33]$. In both cases different approximation techniques have been adopted and hence one cannot expect an identical result.

Gravitational anomalies have a big role to play in the thermodynamics of black holes [4-9,34]. Here I showed that they can reveal the one-dimensional nature of the entropy flow rate through Hawking radiation. This has been established using both the two-dimensional and the four-dimensional anomaly expressions. First the $(1+1)$-dimensional case has been analyzed by the fact that near the horizon any black hole spacetime is effectively two-dimensional; i.e. only the $(t-r)$ sector is important. It was found that the result obtained exactly matches with the one-dimensional photon emission model. Finally the fact is further bolstered by using the four-dimensional anomalous stress tensor in the case of the Schwarzschild metric.

One importance of the present analysis is that the former calculation (i.e. two-dimensional) is applicable to any black holes which are asymptotically flat. This is because, as I mentioned, any static black hole spacetime is effectively of the form (1) near its horizon. Therefore, within this approximation the result (9) is true for any spacetime and hence we can conclude that, in general, the black holes are onedimensional so far as entropy flow is concerned. This information is completely new as the existing discussion $[1,3]$ was confined only to the Schwarzschild metric. Of course, this an approximate analysis and hence only the proportionality constant will vary case by case when the full spacetime will be considered. This happened here also when the analysis was repeated for the four-dimensional case. Moreover, the analysis is completely new, which once again tells that gravitational anomalies have a leading role to play in the thermodynamics of black holes. Let me finish mentioning existing work [35] which discussed a similar topic for the arbitrary $D$ space dimensional Schwarzschild black hole and the black hole in Lovelock gravity. It was found that the dependence of $\dot{S}$ on $P$ is not like the Pendry for Lovelock case, which, as pointed out in [3], is due to an improper choice of the radiat- ing area. Of course, this topic is still open and one needs to further investigate this to achieve any conclusive statement.

Acknowledgments The research of the author is supported by a START-UP RESEARCH GRANT (No.: SG/PHY/P/BRM/01) from the Indian Institute of Technology Guwahati, India.

Open Access This article is distributed under the terms of the Creative Commons Attribution 4.0 International License (http://creativecomm ons.org/licenses/by/4.0/), which permits unrestricted use, distribution, and reproduction in any medium, provided you give appropriate credit to the original author(s) and the source, provide a link to the Creative Commons license, and indicate if changes were made.

Funded by SCOAP S $^{3}$

\section{References}

1. J.D. Bekenstein, A.E. Mayo, Gen. Relativ. Gravit. 33, 2095 (2001). arXiv:gr-qc/0105055

2. L. Susskind, J. Math. Phys. 36, 6377 (1995). arXiv:hep-th/9409089

3. S. Hod, Phys. Lett. B 746, 22 (2015)

4. S.M. Christensen, S.A. Fulling, Phys. Rev. D 15, 2088 (1977)

5. S.P. Robinson, F. Wilczek, Phys. Rev. Lett. 95, 011303 (2005). arXiv:gr-qc/0502074

6. S. Iso, H. Umetsu, F. Wilczek, Phys. Rev. Lett. 96, 151302 (2006). arXiv:hep-th/0602146

7. R. Banerjee, S. Kulkarni, Phys. Rev. D 77, 024018 (2008). arXiv:0707.2449 [hep-th]

8. R. Banerjee, S. Kulkarni, Phys. Rev. D 79, 084035 (2009). arXiv:0810.5683 [hep-th]

9. R. Banerjee, B.R. Majhi, Phys. Rev. D 79, 064024 (2009). arXiv:0812.0497 [hep-th]

10. D.T. Son, P. Surowka, Phys. Rev. Lett. 103, 191601 (2009). arXiv:0906.5044 [hep-th]

11. K. Jensen, R. Loganayagam, A. Yarom, JHEP 1302, 088 (2013). arXiv: 1207.5824 [hep-th]

12. B.R. Majhi, JHEP 1403, 001 (2014). arXiv:1401.1074 [gr-qc]

13. B.R. Majhi, JHEP 1408, 045 (2014). arXiv:1405.4634 [gr-qc]

14. F. Reif, Fundamentals of Statistical and Thermal Physics. McGraw-Hill Book Company, Singapore, p 314

15. B.R. Majhi, arXiv:1110.6008 [gr-qc]

16. K. Srinivasan, T. Padmanabhan, Phys. Rev. D 60, 024007 (1999). arXiv:gr-qc/9812028

17. M.K. Parikh, F. Wilczek, Phys. Rev. Lett. 85, 5042 (2000). arXiv:hep-th/9907001

18. R. Banerjee, B.R. Majhi, JHEP 0806, 095 (2008). arXiv:0805.2220 [hep-th]

19. R. Banerjee, B.R. Majhi, Phys. Lett. B 674, 218 (2009). arXiv:0808.3688 [hep-th]

20. R. Banerjee, B.R. Majhi, Phys. Lett. B 675, 243 (2009). arXiv:0903.0250 [hep-th]

21. S. Ghosh, Int. J. Theor. Phys. 50, 3515 (2011). arXiv:1008.0946 [hep-th]

22. R. Banerjee, B.R. Majhi, Phys. Lett. B 690, 83 (2010). arXiv: 1002.0985 [gr-qc]

23. W.A. Bardeen, B. Zumino, Nucl. Phys. B 244, 421 (1984)

24. L. Alvarez-Gaume, P.H. Ginsparg, Ann. Phys. 161, 423 (1985)

25. L. Alvarez-Gaume, P.H. Ginsparg, Ann. Phys. 171, 233 (1986)

26. L. Alvarez-Gaume, E. Witten, Nucl. Phys. B 234, 269 (1984)

27. J.B. Pendry, J. Phys. A 16, 2161 (1983)

28. P. Candelas, Phys. Rev. D 21, 2185 (1980)

29. R. Balbinot, A. Fabbri, I.L. Shapiro, Nucl. Phys. B 559, 301 (1999). arXiv:hep-th/9904162 
30. B.S. DeWitt, Phys. Rep. 19, 295 (1975)

31. T. Elster, Phys. Lett. A 94, 205 (1983)

32. D.N. Page, Phys. Rev. D 13, 198 (1976)

33. D.N. Page, Phys. Rev. Lett. 50, 1013 (1983)
34. B.R. Majhi, Gen. Relat. Gravit. 45, 345 (2013). arXiv:1210.3306 [gr-qc]

35. B. Mirza, F. Oboudiat, S. Zare, Gen. Relat. Gravit. 46, 1652 (2014). arXiv:1401.0895 [gr-qc] 